\title{
Cultural Heritage Presentation in Virtual Environment: Czech Experience
}

\author{
Jiri Zara, Pavel Slavík \\ Czech Technical University in Prague \\ zara@fel.cvut.cz,slavik@fel.cvut.cz.
}

\begin{abstract}
A permanently increasing graphical power of commonly used computers opens a possibility to present highly detailed and richly textured three-dimensional models of cultural complexes using the web. Utilizing virtual reality paradigms, both experts and ordinary users can explore pieces of cultural heritage in a virtual environment.

This paper introduces three different approaches to cultural heritage modeling and presentation in the Czech Republic. The first example, Celtic oppidum Závist, represents a simple but efficient utilization of the virtual reality for the archaeology. Next project, the Virtual Old Prague, is a complex web-based application allowing dynamic walks through virtual cities. Such models can be theoretically of any size. Finally, the on-going EU project, Virtual Heart of Central Europe, is an example of several cultural heritage sites thematically interconnected into a common virtual environment.
\end{abstract}

\section{Introduction}

Czech Republic has a lot of locations containing objects that are considered to be a part of national cultural heritage. As in other countries round the world some objects like buildings, monuments etc. were destroyed in the past and currently it is impossible to get idea about their former appearance. This fact created an impulse for research in the field of creation of computer models of these objects offering thus to wide audience a possibility to visit these objects in virtual environment.

Another impulse for research of similar type was created by the fact that many locations in the Czech Republic are both of high historical value and aesthetical value - so it is desirable to distribute the information about existence of these sites by means of modern technology (like web). In our research we concentrated ourselves on two main streams: Reconstruction of objects that do not exist anymore and Creation of virtual models of objects (mainly buildings) that carry important information for potential visitors or for people who are interested in history and related disciplines.

\section{Web and virtual reality}

Virtual reality (VR) offers new dimensions for presentation of spatial (3D) objects. One of the main advantages is the possibility to investigate these objects from any viewpoint and at any level of detail in a real time. This sort of investigation is very user friendly because the user behaves in virtual reality in similar way as in a real environment. Moreover virtual reality offers new possibilities that are mostly non-existent in real world (or possibilities that can be exploited with extreme difficulties). As an example we can give situations where the user tries to get impressions that experienced our ancestors many years ago. In [5] a situation is described where virtual model of a cave has been illuminated in the same way as many centuries ago (simulating ancient illumination techniques instead of using modern illumination facilities).

A very important advantage of virtual reality is easy availability of virtual models by means of web. For such purpose, a VRML language is widely used [3]. Besides the feeling of real experience in virtual environment, virtual reality also offers superiority in terms of technical solution - especially from the point of view of volumes of data transmitted. The user has a full freedom to move in virtual reality environment in contradiction to movies where the trajectory has been predefined. But the data volume transmitted in case of virtual models is in orders less than in case of movies.

There are of course some disadvantages of virtual reality technologies in comparison with some other technologies but the advantages prevail in most of applications. As a disadvantage we can consider the necessity to install VR viewer/browser (but in context of other web based technologies available this is not a key problem). The quality of images is in general lower than in case of other technologies but for typical applications this is also not a crucial problem. The beginners might have some problems mastering the navigation tools that are used in typical VR browsers but after short experience this sort of problem vanishes.

In general it is possible to say that technologies based on virtual reality have reached their mature state when they are applicable for tasks of practical use. One of fields of high interest is the use of these technologies in creation of virtual models representing virtual heritage in various countries round the world [1]. 


\section{Simple models}

Territory of the Czech Republic offers a lot of possibilities for archaeological investigations. These investigation help historians (and also common people) to understand the historical development of the territory of the country. One of the most important archaeological findings in the Czech Republic are the Celtic ones. Celts inhabited the territory of the Czech Republic from 500 B.C. up to 100 B.C. Their importance for the future cultural and historical development in this area was immense. They introduced social structure that allowed them to use in effective way natural resources that were at disposal in the area under their rule. The archaeological research in last decades resulted in discovery of remains of several Celtic cities - oppidums. The largest (and the most important one) is Zavist located on the southern edge of Prague.

Celtic oppidum Zavist was established in the first half of the $6^{\text {th }}$ century B.C. For almost two centuries this oppidum was an important location both of social and economic life on the territory of present Czech Republic. The size of the oppidum area was quite large - about $0,27 \mathrm{~km}^{2}$. This area was surrounded by complex fortifications. Besides buildings of various purposes a very important part of the oppidum was created by acropolis with sacral function.

In the framework of cooperation between Archaeological Institute of Czech Academy of Sciences and Computer Graphics Group at Czech Technical University in Prague a decision was taken to perform virtual reconstruction of the acropolis, which was part of the investigated oppidum. This approach (creating virtual models of archaeological sites) attracts more and more attention in last years creating a new discipline: virtual archaeology. Virtual archaeology is a new and rapidly developing area. The main idea is to create the models of the actual artifacts that were found or to make virtual reconstruction that presents the archaeologists' ideas about the appearance of reconstructed sites.

The reconstruction has been based on results of investigations carried out in last decades. Up to now there were only some drawings, created by artists, available that gave some general idea about the structure, size and other attributes of the site. The virtual reconstruction resulted in VRML based model $[6,7]$. Such a model allows the user (virtual visitor) to make virtual walkthroughs in the site. In order to achieve higher quality of static pictures (that might accompany some future texts about the oppidum) the model has been also transformed into 3D Studio Max environment. The pictures that are results of the rendering of such a model are of better quality that pictures generated in web environment.

Currently two other reconstructions of the acropolis on Závist have been created: from the period 500 B.C. and 550 B.C. Both models were used by Czech archaeologists. One of applications to come are pictures in the book under preparation. This book will deal with Celtic history on the territory of the Czech Republic. The project described shows the possibility of wide collaboration between two different disciplines: computer graphics and archaeology.

\section{Complex presentations}

While the previous example represents a standalone, specialized and self-containing set of data, the web offers more complex and flexible approaches to presentation of cultural heritage. One of them is the Virtual Old Prague (VOP) project that has become one of the first serious and professionally designed web applications in the field of web-based virtual cities in the Czech Republic [2]. Former student project has been successfully finished in 2001, then published on the web [4] and made available to Internet users.

The VOP project is the web application that allows walking through a virtual city stored in a remote database. The data are progressively transferred from

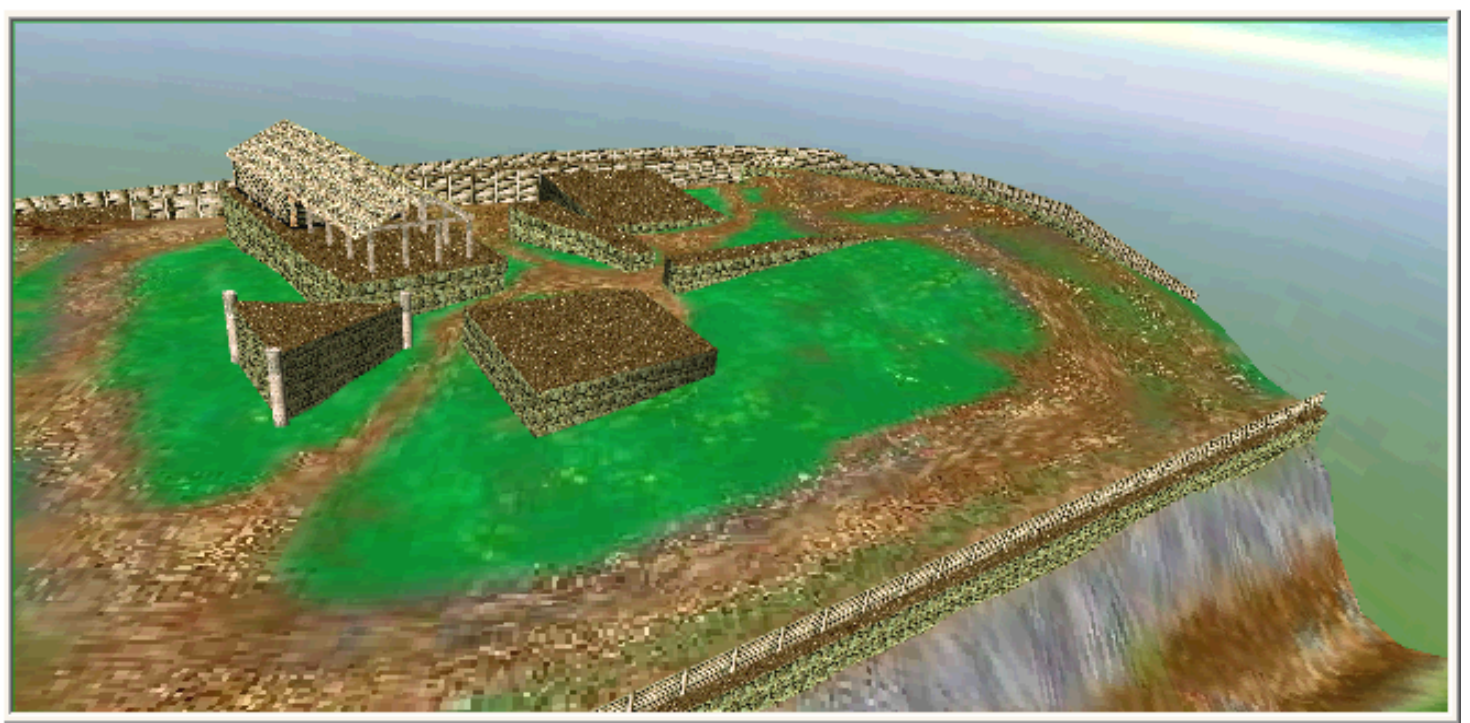

Figure 1. Virtual archaeology - old Celtic site presented in a virtual environment. 


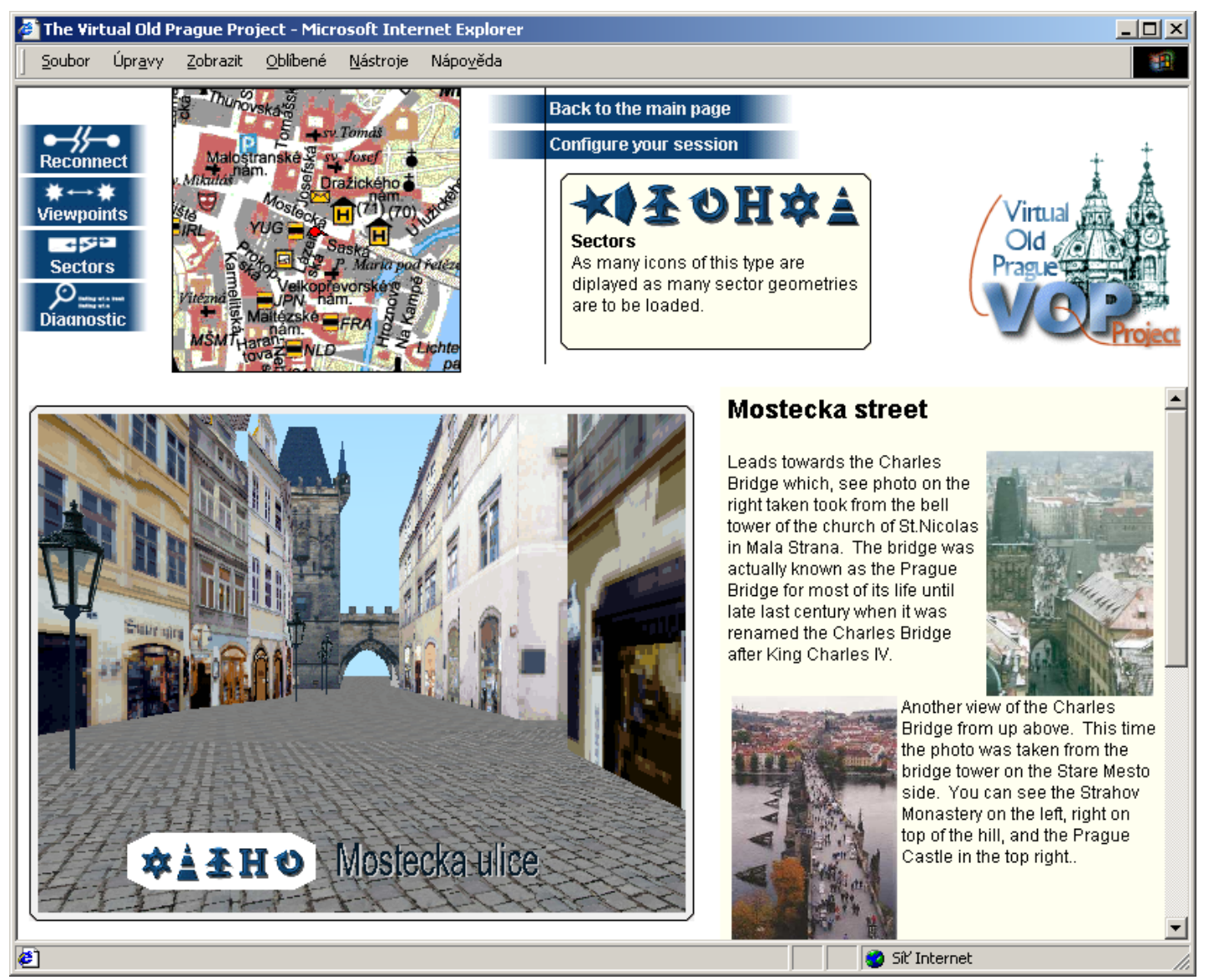

Figure 2. The VOP project represents one of the first complex virtual city presentations on the web.

the server according to a visitor's position. A high frame rate is achieved by rendering visible city parts only. The system has been tuned considering various characteristics - network capacity, computing power on client side, quality of graphics hardware used. Although the overall amount of data describing a virtual city could theoretically grow up to hundreds of megabytes, fine granularity of the city objects (building facades, streets, and other components) helps to achieve smooth and high speed rendering during a walk through the city. Thanks to continuous checking of a user movement, new chunks of data (geometry, textures, etc.) are dynamically requested from the server, while already visited city parts are released from the client memory and moved to a cache. As a result, a client VRML browser has to render almost constant amount of data representing a segment of the city.

The VOP application has been developed strictly on international and industrial standards like VRML, Java, HTML, PHP and SQL database. It should be stressed that the data acquisition process is not automatic but based on manual processing of photos taken from the streets of Prague. Removing perspective distortions, retouching, brightness and contrast tuning, and other raster image transformations represent the most boring work in the preprocessing pipeline. Here we still wait for powerful algorithms from the field of computer vision and reconstruction. The other parts (visibility preprocessing) belonging to the area of computer graphics are computed automatically.
A user interface consists of several parts as seen in Fig. 2. The most informative area is the VRML browser window. Users navigate using a mouse or a keyboard. Their current position is continuously visualized in 2D map using an icon showing a position and orientation. As users walk through the city, both windows (2D map, $3 \mathrm{D}$ view) are updated simultaneously. Moreover, additional information in form of HTML pages (text and images) is synchronously presented in a frame on the right side of the screen.

The VOP application offers three kinds of navigations. The simplest one is a guided-tour. A user selects predefined tour and the application runs without any further user navigation input (3D navigation input is redirected and is taken from the server instead of a mouse/keyboard). It reminds of replaying movie but all VR data are loaded and rendered in a real-time. A user can interrupt the guided tour anytime. The second kind of navigation is based on selecting one of predefined viewpoints. Such viewpoints are shown on sensitive 2D map that can be displayed when clicking on left textual menu. The last navigation mode is interactive walking mode suitable for users experienced in navigation in $3 \mathrm{D}$ virtual space.

Advanced menu contains a lot of settings that help to tune the application performance and to adapt it to user's needs. The VRML window can be enlarged to several predefined resolutions and some frames can be hidden. A suitable VRML browser is able to render the 


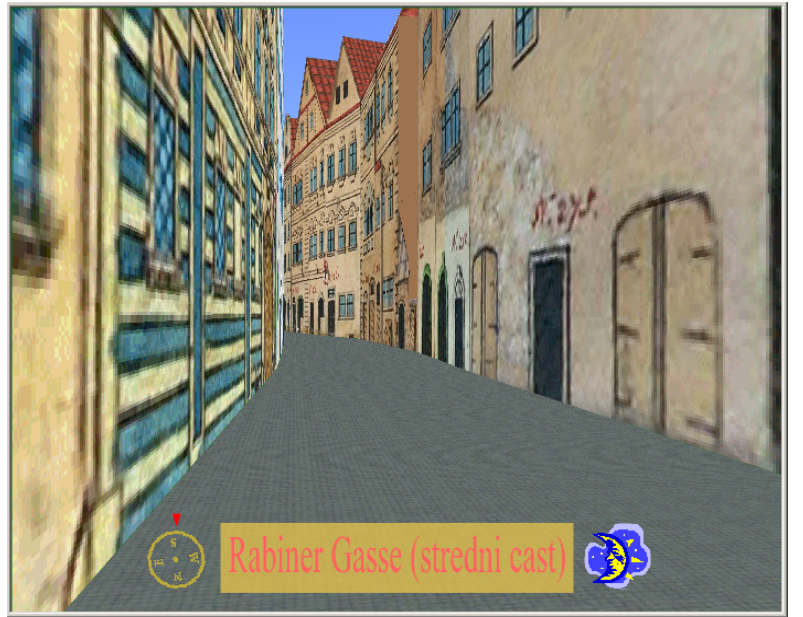

Figure 3. VRML window with a street name and two additional side icons for better navigation.

scene in stereoscopic views thus allowing spatial and highly impressive perception transmitted via Internet.

The project is being further developed towards rich functionality, e.g. search functions followed by an automatic navigation in a virtual space. The software core of the projects is going to be used for other virtual cities under development, namely Bratislava (Slovakia) and Graz (Austria).

Since the client-server architecture is an obstacle for local presentations, we have also simplified a general structure of the VOP application and derived a functional subset that is more flexible and does not require any server. This light version is currently tested for another cultural heritage project, an Old Prague Jewish Town. A preliminary look of the presentation window is shown in Fig. 3. In this case a functional compass that helps to navigate in a $3 \mathrm{D}$ space has enriched a user interface. Another improvement is a possibility to switch between day and night light conditions in a virtual environment (see icon on the right).

\section{Clusters of cultural heritage web based presentations}

Although cultural and historical sites are distributed over the whole real world, a virtual reality together with the web allows wrapping them into one or more thematic envelopes thus highlighting their common and/or different features. This approach is used in the project called Virtual Heart of Central Europe supported by EU Culture 2000 program (A1-CH-SK089).

The main idea behind that project is to present historical architectural gems of four selected cities from different countries - Prague, Graz, Maribor, and Bratislava. Instead of showing well-known tourist attractions only, the project concentrates on vertical solitaires - Towers and Wells. The aim is to digitally preserve carefully selected buildings, to reconstruct them virtually, to prepare a suitable digital storytelling and to publish results on the web using rich hyper linked structures and multi-modal approach.

A big attention has been paid to utilizing a wide range of presentation techniques. While the standard combination of textual and image information creates a solid base for the final web application, threedimensional models are shown in a number of ways. An example of such an approach is shown in Fig. 4, where one of the towers of Prague Charles Bridge is depicted. From the left menu, a user can choose a photo-tour (ordered sequence of images reminding of a walk), panoramic view from the tower (using QuickTime VR movie), full VR model described by means of VRML language, or a computer generated movie with a virtual fly around the model of the tower.

As a representative of another kind of a "vertical" architectural solitaire, a model of the Prague Singing Fountain is shown in Fig. 5. While the left image contains a real photo with many sculpture details, the right image is a snapshot of early VRML model that has
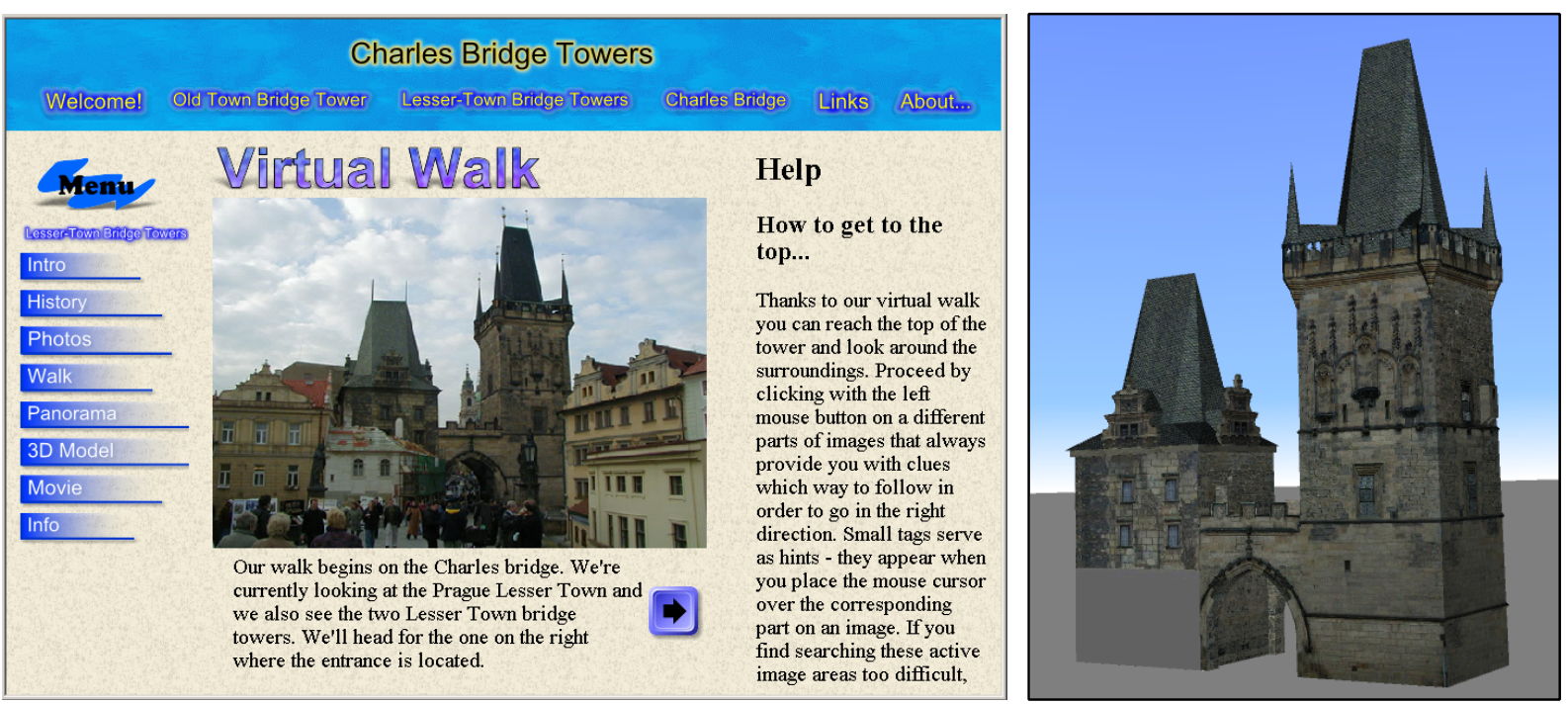

Figure 4. The Charles Bridge Tower in a web presentation (left) and as a VRML model (right). 

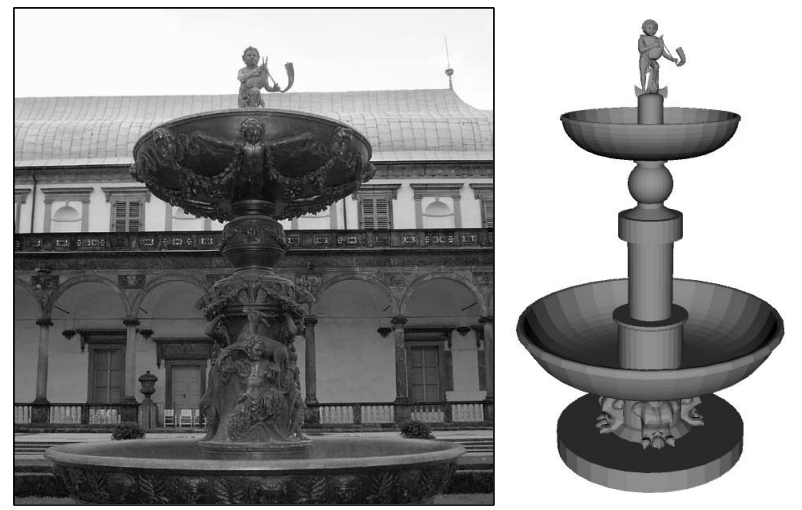

Figure 5. Two presentations of a fountain.

to be still improved using textures and additional geometrical objects.

The wide range of presentation modes meets various users' demands and technical possibilities. It is also a good chance to compare memory requirements for the presentation techniques used. Table 1 gives typical sizes for various kinds of data. All data were compressed and optimized in terms of the best possible quality together with a high compression ratio.

Table 1. Amount of data for virtual tours

\begin{tabular}{|l|c|}
\hline Mode & Typical size of data \\
\hline Set of photos & $1 \mathrm{MB}$ \\
\hline Panoramic QTVR view & $0.5 \mathrm{MB}$ \\
\hline VRML model with textures & $0.5 \mathrm{MB}$ \\
\hline AVI movie & $1 \mathrm{MB}$ \\
\hline
\end{tabular}

Based on our observation, all presentation modes for models of single buildings are of comparable data sizes (i.e. the differences are not very big). If the size is not the issue, users can arbitrarily choose the method they are familiar with.

The Virtual Heart of Central Europe project is scheduled for the years 2003 and 2004. It should bring both novel and generalizing views on the web-based cultural crossroads and meeting points.

\section{Conclusion}

This contribution described experience gained with presentation of cultural heritage of specific type in the Czech Republic. This experience has several aspects. At first the methodology for creating virtual models of architectural monuments has been established. The applicability of this methodology has been proven on several applications of various kinds. At second the existence of projects discussed served as an impulse for cooperation with institutions that have been not aware of existence of these new technologies. These new technologies opened up new ways both for presentation of results achieved (e.g. investigation of new archaeological sites) and for new methods of processing data concerning these buildings of historical value in general. The topic is very attractive nowadays as the distribution of information about cultural heritage serves for better understanding among people with different cultural background. The research in the field offers large possibilities for future research like introduction of multimodal interaction modes (e.g. the a of haptic devices for blind users etc.).

\section{Acknowledgements}

This work has been partly supported by EU Culture 2000 program under the project A1-CH-SK-089 (Virtual Heart of Central Europe) and by the Ministry of Education, Youth and Sports of the Czech Republic under research program No. Y04/98: 212300014 (Research in the area of information technologies and communications). Our thanks belong to our students, Dusan Pavlícek, David Vokoun, Ondrej Pessr, and Michal Rezný, who have prepared virtual models and supplementary data.

\section{References}

[1] J. Willmott, L. I. Wright, D. B. Arnold, and A. M. Day, Rendering of Large and Complex Urban environment for Real Time Heritage Reconstructions, Proceedings of VAST 2001 (Virtual Reality, Archaeology, and Cultural Heritage), Athens, Greece.

[2] J. Zara, Concise Tour to the Virtual Old Prague., EUROGRAPHICS 2002, Short Presentations, Saarbrucken: Eurographics Association, 2002, pages 191-198.

[3] ISO/IEC 14772-1:1997, The Virtual Reality Modeling Language,

http://www.web3d.org/technicalinfo/specifications/vrml97/

[4] Virtual Old Prague Project home page, http://www.cgg.cvut.cz/vsp/

[5] J. Pope, and A. Chalmers, Pre-rendering Acoustics and Illumination for Archaeological Reconstructions, In: Virtual Reality in Archaeology, pages 105-110. ArcheoPress, Oxford, 2000.

[6] J. Krivanek, and P. Slavík, Virtual Reconstruction of Celtic Oppidum Zavist Near Prague In: Spring Conference on Computer Graphics SCCG 2002 - Posters. Bratislava: Comenius University, 2002, p. 56. ISSN 1335-5694.

[7] Virtual reconstruction of Celtic sites, web page, http://www.cgg.cvut.cz/ xkrivanj/projects/celt 